\title{
Nueva arquitectura financiera para el desarrollo en América Latina y Ecuador
}

\section{Nathanaël Legeard ${ }^{\star}$}

\section{Resumen}

Frente a los fracasos o límites de la actual arquitectura financiera mundial (FMI, Banco Mundial, OMC) y regional (CAF, ALADI, FLAR) vigente en América latina, varios países latinoamericanos están formulando propuestas y poniendo en práctica reformas monetarias y financieras con la construcción de una Nueva Arquitectura Financiera Regional (NAFR). La NAFR, tal como se desarrolla en estos momentos, se levanta sobre tres pilares principales: el Banco del Sur (banco de desarrollo), el Fondo Común de Reservas del Sur (fondos para la estabilidad monetaria y de tipos de cambio) y el Sistema Único de Compensación Regional de pagos (organización comercial). El objetivo es alcanzar una mayor autonomía económica y financiera para favorecer un mejor desarrollo sostenible soberano, en un marco de integración regional. Ecuador desarrolla iniciativas nacionales concretas en este sentido.

\section{Palabras clave}

Economía, Arquitectura financiera, soberanía financiera, Banco del Sur, SUCRE, monedas complementarias.

\begin{abstract}
In the face of the failures or limitations of the current world financial structure (FMI, World Bank, WTO) and regional (CAF, ALADI, FLAR), several South American countries are formulating proposals and implementing monetary and financial reforms for the construction of a New Regional Financial Architecture (NAFR). As it's currently developed, the NAFR comprises: Banco del Sur (development bank), Fondo Común de Reservas del Sur (funds for monetary stability) and the Sistema Único de Compensación de pagos(trade organization). The main target is to achieve a greater economic and financial autonomy to promote a better sustaninable development and regional integration. Ecaudor develops specific initiatives in this direction.
\end{abstract}

\section{Key words}

Economy, financial architecture,fFinancial sovereignty, Banco del Sur, SUCRE, complementary currency.

Forma sugerida de citar: Legeard, Nathanaël. 2011. "Nueva arquitectura financiera para el desarrollo en América Latina y Ecuador”. Universitas 14. Enero/Junio. Pp 43-71.

\footnotetext{
* Egresado de Ciencias Políticas de la Univiersidad de La Sorbona-París. nathanael.legeard@gmail.com.
} 
La crisis financiera y económica que comenzó en el otoño de 2008, que ha dado paso a una deflación mundial, resulta en un aumento generalizado del desempleo y una disminución del comercio internacional, y ha reavivado las preocupaciones de reconsiderar el Sistema monetario y financiero internacional. Pero los intentos de cambiar o reconstruir dicho Sistema parecen enfrentarse a un dilema: privilegiar una reforma integral eficaz pero poco probable, o crear iniciativas regionales mucho más pequeñas pero más directamente factibles.

Los países en desarrollo, considerados individualmente, a menudo carecen de medidas efectivas para protegerse y hacer frente a graves crisis financieras y monetarias que puedan afectarles, y que dan lugar a un mayor debilitamiento de sus ya frágiles economías. Esta vulnerabilidad frente a las crisis, para Pedro Páez Pérez, ${ }^{1}$ es el resultado de la "experiencia neoliberal desastrosa" en América Latina, promulgada al principio de la década de los ochenta por el FMI y del Banco Mundial, principlamente, y ejecutada, de manera consentida o no, por los gobiernos de los países de la región. Las políticas neoliberales han llevado a una restricción o destrucción de las estructuras productivas y a un estancamiento económico y financiero que todavía se siente actualmente, y que dejan más vulnerables a estos países frente a las crisis internacionales.

Una respuesta de los países del Sur frente a la inestabilidad monetaria puede ser la creación de fuerzas económicas a nivel regional. En esta perspectiva, varios países de América Latina están formulando propuestas y poniendo en práctica reformas monetarias y financieras, privilegiando una visión y una integración regionales. Así, estos países se están moviendo en la construcción de la NAFR, y desarrollando iniciativas nacionales en respuesta a los intentos de construir una nueva arquitectura financiera internacional, aunque encuentran grandes dificultades para avanzar.

Actualmente se están creando las condiciones para el desarrollo integral de la región, particularmente para proporcionar a los gobiernos de América Latina un mayor grado de libertad en la definición soberana de sus políticas económicas, permitiéndoles contar con instrumentos y mecanismos alternativos y adecuados, tanto financieros como monetarios y comerciales.

1 Pedro Páez Pérez fue Ministro Coordinador de la Política Económica del Ecuador y es el actual presidente de la CENAF, Comisión Ecuatoriana para el diseño de la Nueva Arquitectura Financiera. 
En primer lugar, nos interesaremos en los límites de la actual arquitectura financiera mundial y regional vigente en América latina. En segundo lugar, estudiaremos la propuesta de la NAFR a la escala de varios de estos países. Por fin, presentaremos iniciativas concretas de herramientas financieras para un desarrollo económico y social sostenible en el caso específico de Ecuador.

\section{La actual arquitectura financiera mundial y regional en América Latina: fracasos y límites}

\subsection{Fracasos y efectos perversos de las acciones del Fondo Monetario Internacional y Banco Mundial en América Latina}

A pesar de la independencia política de América Latina ganada hace dos siglos, sus países han seguido viviendo, en gran parte, con dependencia económica ${ }^{2}$ de las potencias del Norte, rompiendo los ideales de los libertadores Bolívar y San Martín en la construcción de la Patria Grande. El siglo XX trajo cambios significativos en la economía capitalista global. Durante los Acuerdos de Bretton Woods, firmados en los últimos momentos de la Segunda Guerra Mundial, surgieron las instituciones que iban a formar los pilares del Nuevo Orden Económico Mundial, diseñados e implementados principalmente a partir de las necesidades y objetivos de las potencias mundiales y de la hegemonía estadounidense. En este marco se crearon las tres instituciones multilaterales, pensadas para ser complementarias, que constituyen la actual arquitectura financiera internacional y que rigen el destino de la economía mundial: el FMI como pilar monetario, el Banco Mundial como pilar del desarrollo, y el intento de la Organización Internacional del Comercio, que será sustituido por el proceso del Acuerdo General sobre Aranceles Aduaneros y Comercio-Organización Mundial del Comercio (GATT-OMC), como pilar comercial.

El FMI es una institución multilateral creada originalmente con el propósito específico de proporcionar préstamos en divisas a corto plazo a los países que enfrenten problemas puntuales en su balanza de pagos, para que puedan man-

2 A través de las exportaciones de materias primas (banano, cacao, madera, petróleo...) bajo un esquema extractivista de depredación de los recursos naturales, principalmente los no renovables. 
tener sus niveles de reservas internacionales, y así preservar la estabilidad de los tipos de cambio de sus monedas. Inicialmente, este papel se entendía en el marco del sistema monetario basado en el patrón oro-dólar y como parte de un régimen con un modelo de tipos de cambio y tasas de interés fijos.

La liberalización de los tipos de cambio, resultado del fin de la convertibilidad de los dólares estadounidenses en oro físico anunciado unilateralmente en 1971 por los Estados Unidos, debiera haber significado la desaparición del FMI, ya que su propósito original se había vuelto obsoleto. Pero, a partir de esa fecha, el FMI se convirtió en una organización motriz en la adopción de un modelo económico capitalista y neoliberal. No solo llegó a ser el último 'salvador' en situaciones extremas sino, sobre todo, fue el que impuso a los países la obligación de llenar una serie de condiciones apremiantes a cambio de entregarles recursos.

Las crisis económicas de los países de América Latina en los años ochenta, debidas a su deuda externa, permitió al FMI, convertido ya en uno de los pilares del Consenso de Washington, ${ }^{3}$ encontrar un terreno fértil para tener éxito en la imposición del modelo neoliberal como opción única de gestión económica. Las condicionalidades del FMI se tradujeron en el hecho de que dos décadas de recuperación económica en América Latina, después de la década perdida de los ochenta, no pudieron ser aprovechadas para mejorar la calidad de vida de las poblaciones vulnerables, a través de un fortalecimiento real de las economías de estos países. Más bien, estos se embarcaron en un proceso de acumulación de reservas y aumento del ahorro interno, a costa de la población (disminución del costo de la mano de obra) y del medio ambiente (explotación de recursos naturales no renovables).

Por otra parte, el Banco Mundial se ajustó a la misma lógica para el financiamiento del desarrollo de las economías más necesitadas. Su función institucional inicial era dirigir sus esfuerzos y canalizar sus recursos para financiar, de la manera más eficaz, proyectos concretos para un verdadero desarrollo económico y social. Sin embargo, en el mismo contexto del Consenso de Washington,

3 Un conjunto de medidas estandarizadas que fueron aplicadas a las economías en dificultades frente a su deuda externa por las instituciones financieras internacionales con sede en Washington (Banco Mundial y FMI) y respaldadas por el Departamento del Tesoro de los EE.UU. 
se convirtió en otro de los mecanismos de condicionalidad para la conversión a una gestión económica neoliberal de los países prestatarios, altamente dependientes de recursos externos para su desarrollo.

El FMI y el Banco Mundial, a pesar de interceder por la transparencia y apertura, nunca adoptaron estas ideas para sí mismos. Sin embargo de ser instituciones públicas, no rinden cuentas a nadie, ni a los ciudadanos que las financian ni a aquellos a los que se supone ayudan. Por último, en cuanto a su modo de gobernanza, estas instituciones internacionales no son absolutamente representativas de las naciones que sirven ${ }^{4}$. Desde los años cincuenta, las intervenciones del Banco Mundial y el FMI en América Latina estuvieron marcadas por las prioridades de la política exterior de los Estados Unidos y de los intereses de la comunidad financiera internacional. Lo que se evidenció especialmente con el apoyo financiero a dictaduras y la reducción de préstamos a gobiernos progresistas. ${ }^{5}$

Por último, el tercer pilar de la arquitectura financiera mundial actual es la OMC, nacida en 1994, sucediendo al GATT (General Agreement on Tariffs and Trade- Acuerdo General sobre Aranceles Aduaneros y Comercio) creado en 1947. El objetivo de la OMC es promover la globalización de la economía y la liberalización del comercio. Esta organización está acusada de favorecer a los contratistas de los países ricos en detrimento de los trabajadores de los países pobres y de acentuar aún más la división internacional del trabajo, sobre todo manteniendo las exportaciones de productos primarios de América Latina. En referencia a la OMC, J. Stiglitz habla de una "hipocresía inmensa: se pretende tener por objeto ayudar a los países en desarrollo, mientras que aquellos se ven obligados a abrir sus mercados a los productos de los países industriales avanzados y, al mismo tiempo, que estos últimos sigan protegiendo sus mercados. Esas políticas son propensas a hacer a los ricos aún más ricos y a los pobres aún más pobres... y más furiosos» (Stiglitz, 2002).

4 Esas constataciones siguen las de J. Stiglitz en su libro Globalization and Its Discontents, W.W. Norton \& Company, 2002.

5 Para una presentación detallada del apoyo del Banco Mundial y del FMI a las dictaduras, ver Eric Toussaint, Banque Mondiale: le Coup d'Etat permanent. L'agenda caché du Consensus de Washington, Liège-Paris, CADTM-Syllepse, 2006. 
Dos décadas de neoliberalismo (ochenta y noventa) han fortalecido las estructuras arcaicas de sumisión económica de América Latina con respeto al Norte. Muchas economías de la región pusieron de relieve procesos de reprimarización de la producción, desmantelamiento de las estructuras productivas existentes, y la concentración de las exportaciones en algunos productos con poco valor añadido y con destino a unos pocos países. También hubo un debilitamiento de las monedas nacionales y del aparato financiero como posible palanca para permitir la inversión productiva. La incertidumbre, que reinaba entonces en todo el continente, incitó las inversiones especulativas en lugar de las inversiones productivas, lo que generó una incapacidad crónica para absorber el crecimiento constante de la población económicamente activa.

Este escenario se ha mantenido hasta principios de la década del dosmil, dejando a los países de la región cada vez más vulnerables a las situaciones de crisis internacionales. Las diferentes organizaciones de la arquitectura financiera internacional que hemos presentado no son, en general, muy favorables a los países en desarrollo y, en particular, a los países de América Latina. Para matizar, nos ocuparemos ahora de las organizaciones financieras en América Latina y especialmente de las que Ecuador es miembro.

\subsection{La arquitectura financiera regional actual: algunas iniciativas, pero insuficientes}

La arquitectura financiera a escala de América Latina también se compone de tres sectores: los bancos de desarrollo para financiar las inversiones productivas y sociales; los tratados o las cámaras de compensación de pagos para el comercio intra-regional; los fondos de cooperación financiera para lograr la estabilidad monetaria y financiera, al apoyar la balanza de pagos y los problemas de liquidez a corto plazo. Nos centraremos en particular en estas tres organizaciones de las que el Ecuador es miembro: CAF, ALADI y FLAR. Estas cumplen muy a menudo papeles complementarios a los de las instituciones internacionales, llenan los espacios de la arquitectura financiera global y proponen servicios financieros que ofrecen una alternativa más grande para los países de la región. 


\section{La CAF: un banco de desarrollo demasiado orientado hacia sus 'clientes'}

La Corporación Andina de Fomento (CAF) es un banco de desarrollo establecido en 1970 y actualmente integrada por 18 países de América Latina, el Caribe y Europa, así como de 14 bancos privados de la región andina. Al principio, era la agencia de crédito de la CAN (Comunidad Andina de Naciones), comunidad que integran cuatro países andinos (Bolivia, Colombia, Ecuador, Perú), y que sucedió en 1996 al Pacto Andino, establecido con el Acuerdo Cartagena en 1969. La CAF fue diseñada como un banco múltiple y una agencia para la promoción del desarrollo y de la integración andina. Los objetivos de la CAF son los de satisfacer las necesidades de los sectores público y privado ofreciendo múltiples productos y servicios financieros a un amplio panel de clientes, compuesto por los gobiernos de los países accionistas, instituciones financieras y empresas públicas y privadas. La CAF promueve el uso de las oportunidades y de los recursos disponibles en su área de acción, mediante la creación de empresas de producción o de servicios, y la expansión, la modernización o la transformación de las ya existentes.

En la información divulgada por la $\mathrm{CAF}$, no se hace referencia explícita al tipo de desarrollo que pretende alcanzar con su acción. Por ejemplo, teniendo en cuenta la situación regional, en ninguna parte se refiere al deseo de reducir las asimetrías entre las economías o la promoción de una distribución de las inversiones en partes iguales entre los beneficiarios regionales. Además, no se indica claramente cómo se canalizan de forma eficaz los recursos de la región hacia su propio desarrollo. No se hace referencia tampoco al desarrollo de prácticas financieras que respeten los principios éticos, la transparencia y la rendición de cuentas. La imagen que presenta la CAF es la de una institución financiera competitiva, orientada hacia el cliente, sensible a las necesidades sociales y que cuenta con un personal altamente cualificado. La CAF evidencia el diseño convencional de una institución financiera que promueve su posición competitiva y su orientación hacia el mercado utilizando el concepto de 'cliente' (CENAF, 2009a).

\section{La ALADI: una organización obsoleta para la realidad del mercado}

La Asociación Latinoamericana de Integración (ALADI) es un organismo intergubernamental, compuesta actualmente por doce países: Argentina, Bolivia, 
Brasil, Colombia, Chile, Ecuador, México, Paraguay, Perú, Uruguay, Venezuela y Cuba. La ALADI sucedió en 1980 a la ALALC (Asociación Latinoamericana de Libre Comercio), nacida en 1960. El objetivo principal fue, a través de la convergencia y la coordinación de los diferentes proyectos de integración existentes, alentar el desarrollo industrial de los países de la región. Se trataba de crear un verdadero mercado común a nivel continental para permitir un mejor uso de los factores de producción, una mejor especialización industrializada de los países comprometidos, una mejor explotación de las ventajas comparativas de cada uno y una ampliación de las oportunidades comerciales. Esto para consolidar las bases económicas de un desarrollo industrial orientado hacia la sustitución de las importaciones, y para fortalecer el poder de negociación de América Latina frente al resto del mundo, tanto en lo económico como en lo político.

La ALALC-ALADI consiguió algunos efectos positivos en el crecimiento del comercio intra-regional entre 1960 y 1979. Pero, en realidad, todos los países querían aprovechar los beneficios de la liberalización del comercio, pero ninguno estuvo realmente dispuesto a abrir sus fronteras y cooperar más estrechamente con sus socios. No lograron crearse vínculos genuinos de complementariedad entre las economías en cuestión. A pesar de los objetivos ambiciosos que podían animar a los países miembros en el momento de creación de la Asociación, el resultado fue más una yuxtaposición de economías que compartían la misma zona geográfica que una verdadera integración regional. Una de las iniciativas interesantes en el seno de la ALADI fue el Convenio de Pagos y Créditos Recíprocos. Con la firma de este Convenio, el sistema de pagos entre los países miembros mejoró y promovió la cooperación formal multilateral entre los bancos centrales de la región, para alcanzar una integración regional en los sectores financieros y monetarios.

El Convenio de Pagos incluye tres componentes principales. En primer lugar un mecanismo de compensación multilateral de pagos del comercio exterior, entre los bancos centrales. Eso permite la reducción de los flujos internacionales de divisas entre los países participantes. En segundo lugar, un sistema de garantía de pagos, que los bancos centrales conceden directamente. Por último, un mecanismo para el reembolso escalonado de los saldos de la compensación multilateral. Después de algunos periodos durante los cuales el Acuerdo fue utilizado de manera significativa, la tendencia actual llega a mínimos históricos. Este deterioro 
en la utilización del mecanismo de la ALADI en el comercio intrarregional se debe a algunas características coyunturales y estructurales.

Entre los factores coyunturales (externos) que impactaron negativamente la utilización del Acuerdo (CENAF, 2009b), se pueden citar la evolución de la globalización de los mercados (internacionalización de los grandes bancos comerciales), el aumento de los riesgos financieros a nivel mundial y la evolución del papel de los bancos centrales (pasando del estatuto de casas principales de cambio al de garantes de la estabilidad monetaria y sistema financiero). Entre los factores estructurales (internos) desfavorables para el Acuerdo, el principal es, probablemente, el uso del dólar estadounidense como medio de compensación y de liquidación de pagos entre bancos centrales. Este uso genera costos de cambio de divisas y de transacción, crea una competencia entre la expansión del comercio dentro de la ALADI o con el resto del mundo (a fin de establecer reservas de divisas para compensar eventualmente los saldos por cobrar para el vencimiento futuro de las compensaciones), y finalmente, no genera un aumento de la liquidez, lo que podría ser beneficioso precisamente durante los periodos de crisis financieras internacionales.

Al final, la ALADI no ha logrado desarrollar significativamente los intercambios intrarregionales, ni una verdadera integración económica regional. Sin embargo, la ALADI puede ofrecer algunas ventajas en el contexto de una posible redefinición de esta entidad en el seno de una nueva arquitectura financiera regional. En particular el hecho de que es un sistema ya establecido, que podría adaptarse a nuevas necesidades o a un nuevo entorno, y el hecho de que el número de países participantes (12) ya es significativo, lo que facilitaría su expansión a toda América Latina.

\section{El FLAR: una institución que debe reforzarse}

El Fondo Latino-Americano de Reservas (FLAR) es una organización financiera internacional. Se trata de un fondo común de reservas de moneda dirigido a la estabilidad financiera de los países miembros, mediante la mejora de su exposición a los riesgos externos y el fortalecimiento de la solidaridad regional. Fue creado en 1978 por los países signatarios del Acuerdo de Cartagena, originalmente llamado el Fondo Andino de Reservas (FAR), y está integrado actualmente por Bolivia, Colombia, Ecuador, Perú, Venezuela, Costa Rica 
(desde 1999) y Uruguay (desde 2008). Los objetivos principales del FLAR son mantener la balanza de pagos de los países miembros otorgando préstamos o garantías de préstamos, mejorando las condiciones de inversión de las reservas internacionales, y contribuyendo a la armonización de las políticas de tipo de cambio, monetaria y financiera de los países miembros. Los dos primeros objetivos son muy similares a los del FMI. Durante el periodo 1976-2003, el FLAR aportó en promedio los recursos equivalentes al $60 \%$ de los fondos de financiamiento excepcional otorgado por el FMI a los países de la CAN.

La ventaja comparativa del FLAR en relación con otras instituciones financieras es su capacidad de respuesta rápida y su flexibilidad para responder a las crisis financieras y de tipos de cambio. Al ser menores las condiciones requeridas, las negociaciones de los préstamos son más rápidas y el desembolso de los fondos más oportuno y más flexible. En cambio, la principal debilidad del FLAR es su capacidad limitada para recaudar fondos. Dado su pequeño tamaño, solo puede proporcionar una ayuda muy puntual a los bancos centrales. El FLAR no puede dar respuesta a una crisis financiera que afecte a toda la región o a varios países, si estos tienen necesidades de recursos al mismo tiempo. Una opción para aumentar su capital sería la de incorporar nuevos países miembros. Su diversificación también sería beneficiosa para el fortalecimiento del FLAR, sobre todo cuando los mercados internacionales se cierran a un solo país o a un pequeño grupo de países con problemas similares.

Otro punto que puede ser cambiado es el hecho de que la mayoría de carteras e inversiones realizadas por el FLAR se realizan en los Estados Unidos, mientras que estos fondos podrían ser invertidos de forma más ventajosa en América Latina. Por otro lado, el FLAR fue la institución responsable de una iniciativa interesante con la creación de los Pesos Andinos, una unidad de cuenta y un medio de pagos internacionales en la región. ${ }^{6}$

Esta ha sido la descripción somera de la arquitectura financiera mundial y regional vigente en América Latina; hemos puesto de relieve una serie de fallos o deficiencias de las instituciones que la conforman. Es precisamente en

6 Para conocer más sobre esta iniciativa, leer el Manual de operaciones y transacciones en Pesos Andinos, FAR, Bogotá, 1987. 
respuesta a esta constatación que se configura, como una alternativa efectiva y soberana en la región, la NAFR, que vamos a analizar a continuación.

\section{La NAFR, para una mejor soberanía económica y financiera}

Frente a los errores o intenciones hegemónicas de las instituciones financieras internacionales y a las carencias o deficiencias de las organizaciones regionales, varios países de América Latina están tratando de volver a dibujar el paisaje de las estructuras financieras con el fin de revitalizar la idea de un desarrollo económico y social soberano y su integración regional. La NAFR es la redefinición de las estructuras tanto financieras como monetarias de la región, basándose en sus características, necesidades específicas y sus propios objetivos. Se trata de conseguir la canalización de los recursos de la región hacia las necesidades de desarrollo que no han sido satisfechas por las acciones de otros organismos multilaterales tradicionalmente destinados a asumir este papel. La NAFR, tal como se desarrolla en estos momentos, tiene como base tres pilares principales: el Banco del Sur (banco de desarrollo), el Fondo Común de Reservas del Sur (fondos para la estabilidad monetaria y de tipos de cambio) y el Sistema Único de Compensación Regional de pagos (organización de comercio).

Los objetivos de la NAFR son romper la dependencia de los países de la periferia con respeto a los mercados financieros internacionales, detener la fuga de capitales, cambiar las prioridades de inversión, canalizar de manera eficiente las capacidades de ahorro y los recursos propios de los países latinoamericanos hacia las prioridades de un desarrollo económico, productivo y social independiente. La idea original era apoyarse en las instituciones ya existentes (CAF, FLAR y ALADI) para redefinirlas y hacerlas evolucionar hacia nuevas organizaciones. En realidad, las nuevas organizaciones se están construyendo en paralelo a las ya existentes, salvo quizá en el caso del fondo de reserva que podría convertirse en el Fondo Común de Reservas del Sur. El desarrollo de cada uno de estos tres pilares sigue una trayectoria independiente y variable dependiendo de los países que los constituyen y de sus prioridades. La ventaja del desarrollo de estas tres organizaciones en paralelo es que son complementarias y se refuerzan mutuamente. 
Las presentaciones sucintas que hacemos más adelante se basan principalmente en la visión y las propuestas de la Comisión Ecuatoriana para la Nueva Arquitectura Financiera regional (CENAF) y de su presidente, Pedro Páez Pérez.

\subsection{El Banco del Sur: un banco de desarrollo de un nuevo tipo}

El Banco del Sur está integrado por siete países latinoamericanos (Argentina, Brasil, Bolivia, Ecuador, Paraguay, Uruguay y Venezuela), y su ambición es evolucionar progresivamente hasta llegar a convertirse en el Banco de la UNASUR. El Banco del Sur es el pilar central de la NAFR, para satisfacer las necesidades de desarrollo de los países de la región. Esta organización quiere ser soberana y excluye cualquier posibilidad de interferencia de elementos externos. Lo que rompe con lo que es casi una norma en el contexto de las estructuras existentes, ya que la participación accionaria de países de fuera de la región es actualmente mayoritaria en los bancos multilaterales de desarrollo. Esta institución se dedica a reducir las desigualdades, la pobreza, la exclusión social y mejorar las capacidades financieras de los países miembros. Por lo tanto, debe ser capaz de aportar soluciones sostenibles a los problemas clave que han retrasado importantes mejoras en los niveles de vida de las poblaciones, respetando los principios de justicia social, respeto al medioambiente, ética, transparencia y rendición de cuentas (control popular y democrático; sus archivos serán de dominio público).

El Banco del Sur establece un conjunto de prioridades de financiación en una nueva perspectiva de desarrollo, con una recuperación de la coherencia económica y productiva en el continente, sobre la base de la financiación de proyectos que alientan cinco soberanías: alimentaria, salud, energética, recursos naturales y conocimientos. La gobernanza del Banco del Sur quiere ser parte de una gestión democrática. En este sentido, a cada país le corresponde un voto. Los destinatarios prioritarios de los préstamos y de las subvenciones son entidades públicas (Estados, provincias, municipalidades y empresas públicas, de producción o de servicios). El Banco otorga la concesión de préstamos con $\mathrm{o}$ sin intereses, $\mathrm{u}$ ofrece ayudas no reembolsables en forma de subvenciones. El Banco del Sur no deberá endeudarse en los mercados de capitales, a diferencia del Banco Mundial y el BID. 
Por otra parte, Ecuador pide que los funcionarios superiores de la institución sean justiciables — si cometen un delito deben ser responsables de él frente a la justicia - y paguen impuestos. Estas medidas reflejan el enfoque promovido por Ecuador: ni economicista ni de negocios; un enfoque que establezca instrumentos económicos que garanticen la aplicación de los derechos humanos fundamentales. El Banco del Sur todavía no está operando. Los órganos legislativos (Asamblea Nacional) de los diferentes países miembros deben ratificar el Tratado Constitutivo para que su funcionamiento sea una realidad. Hasta ahora, solo Ecuador y Venezuela lo han ratificado. En los otros países, las discusiones todavía están pendientes.

Se formaron tres grupos de trabajo que elaborarán los componentes para el funcionamiento operativo del Banco del Sur. Un primer grupo, cuyo país coordinador es Ecuador, reflexiona sobre las orientaciones estratégicas del Banco. Un segundo, cuyo coordinador es Brasil, trabaja sobre temas de gestión financiera, gestión de créditos y gestión de riesgos. El tercero, cuyo coordinador es Venezuela, se dedica a su organización, administración y procedimientos. Se espera que el Banco pueda comenzar sus actividades en el curso de este año (2011). El retraso de su entrada en vigor, que debía ser desde el año 2007, se debe a la agenda específica de cada país y a la falta de voluntad política real de algunos dirigentes, especialmente de Brasil7. Todavía hay algunas pequeñas discrepancias entre las visiones que cada país tiene de este Banco y los tres grupos presentados anteriormente están trabajando precisamente para armonizar las expectativas de los diferentes países. Ahora, vamos a centrarnos en el segundo pilar de la nueva arquitectura financiera regional, que ya opera, a saber, el Sistema Único de Compensación Regional de pagos (SUCRE).

7 Brasil tiende a ver el Banco del Sur más como un posible competidor frente a su propio banco de desarrollo nacional, el BNDES; además considera al Banco del Sur como un instrumento de política comercial y una posible fuente de financiación para dar trabajo a las grandes empresas brasileñas. 


\subsection{EI SUCRE: creación de una moneda electrónica para el comercio regional}

El SUCRE es una iniciativa de los países miembros del ALBA (Alianza Bolivariana para las Américas) para impulsar el comercio recíproco dentro de la región, minimizando el uso de divisas (principalmente los dólares estadounidenses) en los pagos resultantes de esas operaciones. Su creación fue anunciada en abril de 2009 y las primeras operaciones se iniciaron durante 2010. Los países que se adhirieron a este Sistema en 2011 son: Bolivia, Cuba, Ecuador, Nicaragua y Venezuela. El SUCRE es un sistema de pagos internacionales que, a diferencia de los mecanismos convencionales de comercio y de compensación, se basa en una lógica de desacoplamiento relativo al dólar y a la dinámica actual basada en un modelo de división internacional del trabajo articulado en torno a una oposición Norte-Sur. También tiene características distintas a la iniciativa reciente del Sistema de pagos en Monedas Locales (SML), desarrollado entre Argentina y Brasil.

De hecho, el SUCRE se basa en el uso de una moneda electrónica denominada sucre ${ }^{8}$, que permite la canalización eficiente de los pagos internacionales entre los países miembros. Su objetivo es estimular la expansión del comercio sobre la base de una complementariedad productiva como eje de un mejor desarrollo económico, basada en las necesidades y agendas propias de los países participantes. A continuación, el SUCRE buscará alcanzar el equilibrio comercial entre los países miembros, como un mecanismo para reducir las asimetrías y establecer las bases para el desarrollo de nuevos mecanismos de integración regional. Por último, energiza y privilegia los intercambios Sur-Sur. La construcción de la moneda electrónica está respaldada por la configuración de una canasta de monedas locales de los países participantes y de divisas internacionales. A principios de 2011, en las transacciones realizadas a través del SUCRE, el valor del sucre era de 1,25 dólar estadounidense.

8 En referencia al nombre del Libertador Antonio José de Sucre, y a la antigua moneda del Ecuador, cuya economía fue dolarizada en el año 2001. No hay que confundir SUCRE con mayúsculas, que es el Sistema Único de Compensación Regional, y "sucre" con minúsculas, que es el nombre de la moneda electrónica. 
Las funciones básicas del sucre son jugar el papel de una unidad de cuenta común para cuantificar el valor de los pagos internacionales, servir de registro de las transacciones comerciales y ser usado como medio de pago reservado a los bancos centrales que hacen sus pagos entre sí, sin la necesidad de recurrir a divisas extranjeras. Esa última función sustituye a los mecanismos convencionales basados en una compensación en divisas. No se trata de una moneda física ni de un medio de pago al interior de los países participantes; solo se utiliza entre los bancos centrales para canalizar las importaciones y las exportaciones e impulsar el comercio recíproco. Durante una transacción entre un importador en el país A y un exportador en el país B, los dos manejan importes de dinero en sus respectivas monedas locales. Los bancos centrales, a través de la Cámara Común de Compensación, se pagan entre sí en sucres.

Inicialmente, asignaciones en moneda electrónica fueron distribuidas entre los bancos centrales de cada país participante, para proporcionarles liquidez y que puedan hacer sus primeros pagos internacionales. La emisión total inicial fue de 152 millones de sucres. Esta cantidad representa el 20\% de las importaciones de cada país durante el primer semestre de 2010. En función de sus respectivas políticas económicas y comerciales, los países pueden alentar a ciertos sectores de sus economías (gestión administrada) a usar el SUCRE en sus negocios; Sistema que ofrece condiciones más favorables que otras alternativas ya existentes. Desde principios de 2010, varias transacciones se realizaron a través del SUCRE para el comercio de productos como arroz, aceite de soja, llantas... por importes de transacciones de hasta 4’240.000 sucres (el equivalente de 5,3 millones de dólares estadounidenses).

Los beneficios del SUCRE son múltiples. La utilización de una moneda electrónica genera liquidez para los bancos centrales a partir de su esencia fiduciaria. Se aumenta la capacidad de importación de los países participantes, a través de las asignaciones en dinero electrónico como activos de reserva. Se trata de un instrumento importante para promover un aumento en el comercio recíproco. Luego, el Sistema reduce los costos del tipo de cambio al no utilizar el dólar u otra divisa como intermediario para los pagos internacionales. También minimiza los costos de transacción al no usar largas cadenas de correspondencia entre las instituciones financieras. Así, el SUCRE puede aumentar los márgenes de beneficio para los actores del comercio exterior. 
Para que este Sistema sea sostenible, su uso debe ser la mejor alternativa frente a otros medios convencionales de pagos internacionales (costos de transacción reducidos, compromiso de los bancos centrales). Habrá que llegar a que los países participantes se comprometan formalmente a aceptar el dinero electrónico como único medio de pago para sus transacciones. Por último, estos países deben realmente tener éxito en la coordinación de la gestión de sus políticas monetarias y de tipos de cambio. Para concluir, el SUCRE se presenta como una propuesta viable de nuevo tipo, bajo los principios de la NAFR, que incluye algunos elementos que ya existen en sistemas de pago vigente, pero que vuelve a definirlos en combinación con nuevas iniciativas técnicas.

\subsection{El Fondo Común de Reservas del Sur: para una mejor canaliza- ción de las reservas monetarias}

El tercer pilar de la NAFR es el Fondo Común de Reservas del Sur, que tiene como objetivo promover la estabilidad monetaria y los tipos de cambio de los países de la región. El objetivo es diseñar herramientas que permitan mantener las reservas monetarias a un nivel adecuado, para afrontar en caso de necesidad los efectos de posibles crisis financieras locales o internacionales. La visión presentada a continuación es la de la CENAF. El estado actual de la propuesta de creación de un Fondo Común de Reservas en el Sur, por parte de la Comisión Ecuatoriana, es una modificación y una redefinición del papel del Fondo Latinoamericano de Reservas (FLAR) ya existente, más que la creación de una nueva organización.

Actualmente, los bancos centrales, de forma individual y autónoma, mantienen altos niveles de reservas, parte importante del ahorro nacional del país, para responder a posibles crisis o ataques especulativos. El resultado es una inmovilización de capitales que, invertidos en el extranjero con rendimientos muy bajos, de acuerdo con los principios prioritarios de seguridad y de liquidez, impiden el cumplimiento de las necesidades específicas de financiación del desarrollo local. Estos recursos invertidos en el Norte favorecen el desarrollo del primer mundo, mientras que para su propio desarrollo, los países de la región deben hacerse financiar mediante créditos importados a través de instituciones multilaterales con todas sus condicionalidades. Esta situación paradójica 
es el resultado de la presión continua que soportan los bancos centrales para disponer de liquidez suficiente y pagar el servicio de la deuda externa. Este mecanismo de acumulación deriva de las condicionalidades impuestas por el FMI para obtener préstamos de su parte. Por eso, los bancos centrales acumulan excesivas reservas internacionales en comparación con las necesidades reales de los países.

Sería posible lograr una economía de escala y una reducción de los costos administrativos si, en lugar de que cada banco central gestionara sus propias reservas, el FLAR fuera el responsable de la gestión de un fondo común o de un pool, que comprendería la mayor parte de las reservas de cada uno de los países. Esto podría ser posible ya que las directivas de inversión de los bancos centrales y las del FLAR son muy similares actualmente. Otras propuestas podrían ser la aplicación de garantías de reservas entre los bancos centrales o el desarrollo de mecanismos que permitan la creación de un mercado continental de valores monetarios. Eso permitiría, sin dejar de lado los mecanismos ya vigentes en el FLAR, incorporar estos nuevos elementos, lo que constituiría una redefinición del papel actual del FLAR. El objetivo es, en este sentido, establecer una nueva red y nuevas relaciones entre los bancos centrales. Se trata de herramientas que permitirían a los bancos centrales contar con sistemas de seguridad adecuadas para afrontar los diversos problemas, sin tener que mantener altos niveles de reservas, colocados fuera de la región, y que podrían buena parte de ellas ser canalizadas hacia la financiación del desarrollo local.

Todo eso podría proporcionar a los bancos centrales de los países participantes la posibilidad de mantener mecanismos de disuasión importantes, lo que en sí mismo constituiría un primer elemento para prevenir ataques especulativos y crisis financieras. Por último, en varios países de la región a veces es criticado el hecho de tener que delegar la gestión de las reservas nacionales a organizaciones multinacionales privadas. Si el FLAR consigue fortalecer sus servicios y ser más eficiente en la gestión de las reservas, muchos bancos centrales probablemente encontrarían que da mejor imagen política delegar la gestión de sus activos internacionales a una organización regional pública. 


\subsection{Beneficios adicionales de la NAFR en el caso de Ecuador}

Ecuador, por tener una economía dolarizada, es probablemente el país en la región que más necesita de estas nuevas instituciones que se presentan en el marco de la NAFR. Los dólares estadounidenses que utiliza Ecuador para comprar productos extranjeros o pagar las transacciones hacia el exterior desaparecen de la circulación en el interior del país. Si el país no logra, al mismo tiempo, mantener un flujo suficiente de dólares que ingresen al país a través de las exportaciones o de las remesas de los emigrantes, el volumen de la masa monetaria disminuye y restringe la liquidez interna de la economía ecuatoriana, lo que puede tener graves efectos sobre la estabilidad del sistema financiero nacional.

Estos problemas de liquidez, que se dejan sentir tanto en las empresas micro, pequeñas y medianas como en los grandes negocios, plantean la necesidad de crear mecanismos para reducir la utilización de las divisas extranjeras (dólares estadounidenses), recuperar un conjunto de funciones de la soberanía nacional en la gestión de la economía, y aumentar el margen de maniobra en el desempeño de la política económica. Esto proporcionaría las condiciones necesarias para una mayor estabilidad y seguridad en la gestión macroeconómica de un país dolarizado como Ecuador. Las crisis financieras, las caídas de las exportaciones y la imposibilidad de influir en el valor del tipo de cambio, mientras los países vecinos y competidores de Ecuador en algunos productos pueden devaluar sus monedas, constituyen grandes dificultades cuando se trata de garantizar un ritmo de exportaciones que permita compensar el aumento de las importaciones. Al mismo tiempo, la actual crisis reduce las oportunidades de empleo para los inmigrantes ecuatorianos en el Norte, lo que reduce el importe de las remesas y la inyección de divisas extranjeras (dólares estadounidenses), lo que contribuye a configurar un escenario de incertidumbre en cuanto a la capacidad de mantener la liquidez interna de la economía ecuatoriana.

Por eso, en paralelo al desarrollo de la NAFR, es necesario completar estas iniciativas con el desarrollo de otras para una nueva arquitectura financiera nacional y apoyar otro tipo de desarrollo más democrático, descentralizado y sostenible. En ese sentido, nos ocuparemos ahora de las iniciativas específicas que se están desarrollando en Ecuador y que prolongan, de manera concreta, los principios que han sido presentados a través de las instituciones que conforman la NAFR. 


\section{Las iniciativas de reapropiación de las herramientas finan- cieras para un desarrollo económico y social sostenible en Ecuador}

La NAFR, presentada hasta ahora, debe ser capaz de desarrollarse en paralelo y en interacción con una nueva arquitectura financiera doméstica (o nacional). Economías como la de Ecuador se convirtieron o mantuvieron en la periferia. Desde hace más de dos siglos se desarrollaron estructuralmente en el marco de una división internacional del trabajo, que convirtieron a los países del Sur en productores obligados de materias primas. Básicamente, lo único que garantiza un espacio viable para estas economías es la devaluación de la fuerza de trabajo y la explotación de recursos naturales no renovables.

Otra problemática es el hecho de que una gran parte del ahorro en las zonas más pobres de Ecuador, capturado por las instituciones financieras, termina en los mercados financieros de las principales ciudades del país (Quito, Guayaquil y Cuenca) o, aún peor, es exportado hacia los mercados extranjeros; en su mayoría, el dinero no está reinvertido en las comunidades de donde proviene. Los bancos que captan los dólares de una comunidad vuelven a inyectar solo una parte mínima del importe en la misma. Por ejemplo, de los 18 mil millones de dólares de depósitos populares en el Ecuador, más de 5 mil millones están colocados fuera del país, especialmente en Estados Unidos. ${ }^{9}$ Así, los más pobres en Ecuador ayudan a financiar las guerras en Irak y Afganistán... Entonces, es necesario tener en cuenta soluciones prácticas a estos problemas específicos. Parece imperativo crear, por ejemplo, nuevas formas de dinero. Dinero que no sea utilizable para la acumulación, la especulación y la asfixia de las economías. Una moneda que dinamice el deseo y capacidad real de las personas — cuyos

9 En Estados Unidos, que tienen uno de los mercados de crédito más desarrollados, las carencias en el reparto de créditos - las minorías, las regiones desfavorecidas - fueron consideradas tan graves que adoptaron en 1997 el Community Reinvestment Act (CRA), que obliga a los bancos a conceder préstamos a los estratos sociales y zonas geográficas menos favorecidas. Más exactamente, los bancos tienen que reinvertir localmente (en forma de préstamos) al menos el 50\% de los ahorros que los depositantes locales les entregan. El CRA ha sido una herramienta importante, aunque controvertida, para alcanzar objetivos sociales clave. 
proyectos estuvieron limitados por falta de liquidez - a trabajar y participar en el aparato productivo.

La cuestión del dinero es un tema central, que a nivel micro debería contribuir a establecer mecanismos de recirculación y reciclaje de los recursos domésticos en la producción local. Parece indispensable crear mecanismos de apoyo a la economía popular y solidaria. La escasez de dinero y su mala distribución es un efecto recurrente causado por la antigua arquitectura financiera. Nuevas iniciativas y avances en términos monetarios abren perspectivas interesantes. Varios de estos sistemas alientan el uso de los recursos a nivel local, tratando de reducir el uso del dólar estadounidense, porque los dólares que se utilizan para comprar afuera son dólares que dejan de circular en las economías locales. En respuesta a esta situación, existen en curso varias iniciativas y alternativas en Ecuador, de las que nos vamos a ocupar a continuación.

\subsection{Dos iniciativas del Banco Central del Ecuador (BCE) con el uso de tecnología de la información}

El BCE está ejecutando actualmente dos proyectos importantes, cuyo objetivo es restringir el uso del dólar para reducir la presión sobre esa moneda y recuperar algo de soberanía monetaria y financiera, en gran parte perdida debido a la dolarización de la economía del país en 2001.

\section{Los pagos a través de teléfonos móviles: una herramienta para el desa- rrollo socioeconómico}

El BCE está trabajando desde hace algunos años para poner en práctica un Sistema de Pagos Móvil (dinero transferible por teléfono móvil). Este proyecto se ha trabajado con varias organizaciones de los sectores público y privado. El BCE observó que en el sistema financiero tradicional hay numerosas personas sin ningún acceso a servicios financieros básicos..$^{10}$ Las empresas financieras privadas (bancos, compañías de seguros...) y la dinámica económica actual las

10 Solo el $65 \%$ de los ecuatorianos tienen acceso una cuenta bancaria, lo que excluye al $35 \%$ de la población total. 
han vuelto invisibles. El objetivo del proyecto de Pagos Móviles es volver a hacer visibles a estas personas y generar cambios en el sector financiero. Mientras que la industria financiera tradicional no ha logrado satisfacer al conjunto de la población, ahora la industria de las telecomunicaciones está desarrollando una cobertura casi en la totalidad del territorio ecuatoriano (más del $90 \%$ de la población). Por lo tanto, se hace necesario borrar los límites de la industria financiera y, gracias a las telecomunicaciones, facilitar el acceso de toda la población a los servicios financieros.

Así, el teléfono móvil fue identificado como una posible herramienta de la política económica, que puede ser utilizado como un medio complementario de pago. Esto permite desmaterializar el dinero reduciendo, al mismo tiempo, la necesidad de usar el papel moneda y la presión sobre el dinero físico. ${ }^{1111}$ El Sistema de Pagos Móviles tiene como objetivo establecer un medio de transacción monetaria segura, a coste reducido y con cobertura nacional. El Sistema está garantizado por el BCE y utiliza las plataformas de los operadores de telefonía para ofrecer cobertura en todo el país. Este sistema convierte al teléfono móvil casi en un cajero automático o en una billetera virtual para las personas que no tenían acceso a una cuenta bancaria. Las personas, independientemente del operador telefónico utilizado, pueden en tiempo real, enviar y recibir dinero, comprar bienes, pagar facturas y recibir subsidios estatales a través de su teléfono móvil, incluso el más básico. Todas las transacciones se realizan por SMS, a coste de SMS.

El Sistema de Pagos Móviles puede transformar la vida de las personas de bajos ingresos. Por ejemplo, los empleados que trabajan en las principales ciudades (Quito, Guayaquil y Cuenca) podrán transferir remesas a sus familias que viven en las comunidades rurales, de manera muy sencilla y a un precio muy bajo. El BCE, en coordinación con el Ministerio de Telecomunicaciones y la SENATEL (Secretaría Nacional de Telecomunicaciones), está generando las condiciones necesarias para poder implementar el Sistema de Pagos Móviles a escala nacional.

11 Es fácil darse cuenta de la escasez física de dinero en la vida cotidiana en Ecuador: muy a menudo, por ejemplo, los pequeños comerciantes no pueden devolver el cambio a los clientes que quieren pagar con billetes, incluso billetes pequeños (5, 10 o 20 dólares). 
El BCE también debe facilitar y proporcionar una red de centros de distribución y de operaciones, donde la gente pueda realizar transacciones a bajo costo y operaciones de pago y de retirada de dinero físico. Por último, el BCE debe cumplir un trabajo importante para sensibilizar a la gente en el uso de esta herramienta móvil y generar un nivel de apropiación de esta tecnología. El proyecto piloto se lleva a cabo este año a través de la red del Grupo Social FEPP (Fondo Ecuatoriano Populorum Progressio), que ya cuenta con 365 centros de gestión a nivel nacional. No se trata de una nueva moneda, solo es la forma virtual del dólar estadounidense. De ninguna manera se va a cambiar el dólar como unidad de precio y de cuenta. Es un sistema como el de las tarjetas de crédito. No se trata de romper con la dolarización (preocupación de muchos ecuatorianos); sino, más bien, de fortalecer el sistema financiero existente.

\section{FACTOREPO: Sistema público de compensación de facturas}

Una segunda iniciativa emanada del BCE es un sistema público de factoring: el proyecto Factorepo, un sistema de compensación de facturas que inició su desarrollo y ejecución en 2009. Fue desarrollado con el propósito de ayudar y apoyar a los pequeños productores y empresarios, en reducir sus necesidades de liquidez. El sistema combina las facturas que se emiten entre un comprador y un vendedor en una transacción específica; luego, el sistema emite bonos virtuales equivalentes al valor de las facturas. De esta manera el receptor de los bonos recibe inmediatamente liquidez, ${ }^{12}$ lo que da flexibilidad y facilidad a las transacciones y a las actividades productivas. La misión del proyecto Factorepo es fortalecer el sector productivo del país y estimular la economía nacional interna. Gracias a la compensación, al financiamiento y a los pagos de las cuentas por pagar y por cobrar de las empresas del sector productivo nacional; la idea es generar un sistema alternativo que contribuya a la promoción de una nueva estructura financiera del país, permitiendo incluir la participación de la mayor cantidad posible de actores. El BCE es el administrador del sistema y utiliza Internet como la herramienta principal para hacerlo.

12 Al contrario de las facturas convencionales que se pueden pagar dentro de 30 o 90 días. 
Si uno de los participantes, por ejemplo, tiene más clientes que proveedores, tendrá un superávit de los medios internos de intercambio (los bonos). Aquellos que necesitan temporalmente bonos pueden hacer un factoring de sus cuentas por cobrar. El sistema puede proporcionar bonos a los participantes que tienen cuentas por cobrar, es decir, a los que están a la espera de recibir un pago, de tal manera que puedan pagar a sus proveedores más rápidamente. La cantidad de bonos en el sistema es proporcional a la cantidad y al importe de las cuentas por cobrar de todos los participantes.

En una encuesta de opinión realizada entre 1.500 empresas ecuatorianas, se determinó que el $76 \%$ de ellas no liquidan directamente sus facturas y solo el $24 \%$ utilizan una u otra forma de compensación de facturas. Lo que demuestra la existencia de un gran mercado potencial, que desconoce hasta ahora el uso que se puede hacer de un sistema de factoring, como el sistema Factorepo. Al final, este sistema proporcionará un nivel de flujos de moneda reducido (en efectivo, en divisas, de tesorería) y habrá menor presión sobre el dólar.

Ahora vamos a estudiar la experiencia descentralizada de las cooperativas de ahorro y crédito que forman parte de la economía social y solidaria, y dar una mirada a los proyectos de creación concreta, esta vez, de monedas alternativas y complementarias.

\subsection{Cooperativas de ahorro/crédito y monedas alternativas y complementarias}

\section{La Red Nacional de Finanzas Populares y Solidarias del Ecuador (RENAFIPSE)}

La RENAFIPSE nació de un trabajo directo de varios años a nivel local, apoyado y alentado por las Estructuras Financieras Locales (cooperativas de ahorro y crédito, cajas y bancos comunales). Se trataba de fomentar una ideología de red, compañerismo, trabajo, apoyo y aprendizaje mutuo para ser más eficientes y competitivos frente al poder de los bancos; con la creencia profunda en el desarrollo local orientado hacia la consolidación de proyectos productivos.

La RENAFIPSE se convirtió en la única red nacional, que reúne a las regionales, que a su vez agrupan a un gran número de instituciones financieras locales. La RENAFIPSE basa su trabajo en algunas prioridades: incidir en las 
estructuras institucionales, políticas, sociales y económicas de Ecuador; ayudar a la creación de un modelo de desarrollo económico más equitativo; apoyar la formación y la educación; promover la adquisición de tecnología apropiada para las estructuras financieras locales; apoyar el fortalecimiento de los procesos de gobernanza de cada institución y fomentar el desarrollo de alianzas estratégicas entre los actores de la economía social, las instituciones privadas, las ONG y las instituciones gubernamentales del Estado.

La RENAFIPSE, con el apoyo técnico de la Fundación Pachamama y de la Fundación STRO, decidió promover la aplicación de sistemas monetarios complementarios en sus Estructuras Financieras Locales (EFL) para aumentar la participación de cada uno como gerentes principales del desarrollo económico local en las comunidades donde realizan sus operaciones. Las finanzas populares y solidarias son un conjunto de alternativas económicas que alientan el desarrollo sostenible y que refuerzan el vínculo entre las redes de desarrollo económico y social. Se favorecen los ahorros para poder usar estos recursos en el financiamiento de actividades productivas. Las tasas de interés de los préstamos están reducidas, y las de ahorro se ofrecen a tasas más altas. El crédito no es la única solución para desarrollar los pueblos. Antes de conceder préstamos a las personas, también es importante enseñarles a ahorrar.

En Ecuador, el modelo de producción y comercialización de las cooperativas de acopio y de los puntos de venta ha sido un éxito debido en gran parte al hecho de que más de la mitad de sus miembros son agricultores. Así, además de ser productores, son también consumidores en las cooperativas. Este modelo no incita a que lo mejor de la producción de los agricultores sea exportado inmediatamente. El punto de partida es conseguir que las familias de los campesinos estén bien alimentados; luego, se piensa a escala del barrio, después se va ampliando el alcance de la acción hasta, finalmente, considerar la eventualidad de exportar. Hay que resaltar en este modelo cómo se privilegian las relaciones con sus gobiernos locales, de que los procesos no sean aislados y cuenten con la participación de varias organizaciones que tienen un cierto poder económico, político o social; todos estos son factores de motivación y confianza para sus miembros. 


\section{Sistema de Intercambio Solidario y creación de monedas locales}

Este proyecto es una iniciativa del sector de la economía popular y solidaria, basada en métodos monetarios alternativos, con el fin de promover el desarrollo económico local en las zonas donde se establecen lazos financieros y sociales entre las empresas y sus residentes, a través del fortalecimiento de sus vínculos comerciales. El Sistema de Intercambio Solidario tiene como eje fundamental la creación de redes locales que reúnen empresas comerciales, unidades productivas e iniciativas de empresarios autónomos, que aceptan y utilizan un medio de pago local — una orden o un bono-, llamado UDIS (Unidad De Intercambio Solidario), para la compra de bienes o servicios. Se trata de medios de intercambio locales independientes que permiten generar un poder de compra estable y sostenible en el seno de las comunidades locales.

Los sistemas monetarios complementarios son medios de pago emitidos por una institución, una comunidad, un grupo de personas o empresas, etc. Circulan dentro de un área geográfica determinada y de un grupo específico, definido y limitado. Están respaldados por la capacidad de producción, los inventarios y las ventas de los participantes. Es importante señalar que no eliminan la moneda nacional, pero la complementan. Estos sistemas se caracterizan por el hecho de facilitar los intercambios donde hay una escasez de dinero y de evitar la acumulación. Son creados para equilibrar y conectar la oferta y la demanda local de bienes y servicios. Tienden a ser menos vulnerables a los avatares de la economía internacional y menos dependientes de los préstamos bancarios externos. En numerosas ocasiones, el dinero sale muy fácilmente de las economías locales (comunidades), pero no regresa y no entra con la misma facilidad. El uso de un medio de intercambio local genera un poder de compra que no puede 'escaparse' de la zona geográfica, o tarda más en salir.

\section{Ejecución del proyecto}

La RENAFIPSE, con el apoyo y los consejos de la Fundación Pachamama y de la Fundación STRO de los Países Bajos, decidió llevar a cabo proyectos piloto para promover los sistemas monetarios complementarios a través de las cooperativas de ahorro y crédito, filiales de la red nacional. Después de un proceso exhaustivo de selección, tres cooperativas filiales de la RENAFIPSE fueron elegidas: Coopera, Intégrale y San Miguel de Sigchos. Estas socias te- 
nían las condiciones más adecuadas para impulsar el desarrollo de métodos monetarios alternativos en el seno de las comunidades donde operan. El objetivo era demostrar que existen otras soluciones técnicas y culturales para crear riqueza local. Los proyectos que se implementan están diseñados sobre la base de las metodologías desarrolladas y aplicadas por la Fundación STRO en los últimos años. Dos métodos principales se utilizan — conocidos por sus siglas en inglés-: la CBC (Commodity Backed Currency) y la LBC (Loan Backed Currency).

El primero, $\mathrm{CBC}$ (moneda respaldada por materias primas), es un método según el cual una organización (una empresa asociativa, una cooperativa de consumidores, una cooperativa de productores) paga a sus proveedores, empleados o afiliados en una moneda propia, que está garantizada por el inventario de las provisiones o por la capacidad de producción de la empresa emisora. En general, el medio de intercambio es un bono. Por ejemplo, una cooperativa de productores de azúcar puede pagar a sus proveedores (productores de caña de azúcar), una parte con bonos. Los productores utilizan los bonos para comprar en los negocios locales. Los negocios locales utilizan a continuación los bonos para comprar el azúcar de la cooperativa. Los beneficios para la cooperativa son, en primer lugar, reducir sus costos de financiamiento, mediante el aumento de su fondo de operaciones sin tener que pedir préstamos y, en segundo lugar, asegurar sus ventas ya que a cada bono emitido corresponde un seguro de venta futura.

El segundo método, LBC (moneda respaldada por créditos), tiene como objetivo estimular la economía local y el desarrollo social de las comunidades a través de un aumento de su liquidez, a través de la circulación local de bonos de crédito, emitidos y administrados por organizaciones de microfinanzas, como las cajas rurales o las cooperativas de ahorro y crédito. Los bonos emitidos son garantizados por deudas que a su vez se aseguran a menudo sobre bienes. Los bonos pueden ser utilizados para comprar productos locales o para pagar salarios. La ventaja para las instituciones de microfinanzas es que pueden aumentar su capital de préstamos, sin ningún costo financiero adicional. Especialmente en los países donde las tasas de interés son altas, este método puede causar grandes diferencias en las tasas de interés entre los préstamos en moneda nacional y los préstamos en forma de bonos (unidades internas de las comunidades). 
A continuación se realiza una descripción del diseño del proyecto planteado para cada cooperativa de ahorro y crédito, mencionando ciertos aspectos generales y algunas ventajas de la implementación de estos modelos en las comunidades locales. En el caso de la cooperativa de ahorro y crédito Coopera, se aplicaron los dos métodos: el método CBC para los Centros de Acopio y Comercialización de la cooperativa; y el método LBC para las operaciones financieras propias de la cooperativa. Ambos métodos se aplican combinados para obtener mejores resultados en la emisión de bonos y tener garantías más sólidas. Para llevar a cabo la fase piloto y dar a conocer el proyecto, la emisión del bono se realizó solo con los miembros productores de la cooperativa, de forma individual. Se les ofreció entonces la oportunidad de recibir estos bonos como medios de pago, que entonces podían utilizar en los centros de comercialización Coopera, así como en las tiendas afiliadas a la red. De esta manera, el proyecto se centró en promover una mejor re-circulación y reciclaje de los recursos locales. Posteriormente, se analizarán otras estrategias de circulación de los bonos.

Para la cooperativa de ahorro y crédito Intégrale, fue diseñado un sistema de bono que conlleva las principales actividades productivas de la parroquia de $\mathrm{Si}$ nincay (ubicada en el sur de Ecuador, cerca de la ciudad de Cuenca); por ejemplo, los artesanos que fabrican ladrillos y tejas pueden tener acceso a préstamos nuevos en forma de bonos, que pueden ser utilizados para el pago de mano de obra o para comprar materiales. Para esto, hay que trabajar en la conformación de una red de empresas y negocios locales que aceptan los bonos, dentro de la cual hay que incluir, en primer lugar, a los proveedores de materiales de los artesanos; en segundo lugar, a otros negocios y empresas, y posteriormente a las iniciativas productivas y de auto-empleo a nivel local. La cooperativa recibe los bonos como medio de pago para el reembolso de los préstamos y para pagar los intereses de los préstamos otorgados a sus miembros.

La cooperativa de ahorro y crédito San Miguel de Sigchos (en el centro del país) ha encabezado la creación de un sistema de intercambios solidarios sigchense como una estrategia para fortalecer la economía local, pero también con el objetivo de desarrollar el sentimiento de pertenencia al cantón Sigchos por parte de sus residentes. El proyecto fue diseñado para proporcionar a los miembros de la cooperativa la posibilidad de acceder a préstamos en forma de 
bonos, reduciendo los costos financieros y con la posibilidad de utilizar esos bonos en los negocios locales, disfrutando de los beneficios que las empresas ofrecen a los consumidores miembros del sistema. También tiene como objetivo aumentar las ventas de los negocios participantes, por el hecho de que muchos miembros que reciben bonos se convertirán en nuevos clientes de estos. El Sistema de Intercambio Solidario está totalmente financiado y garantizado por la cooperativa de ahorro y crédito San Miguel de Sigchos, que es la institución encargada de vigilar y supervisar las distintas operaciones realizadas con los bonos.

Estas tres cooperativas de ahorro y crédito demuestran que es posible la creación de bancos alternativos que fomenten otro tipo de producción que el que promueven los bancos tradicionales. Estas cooperativas permiten ofrecer servicios financieros a comunidades que son habitualmente excluidas del sistema financiero convencional. Estas iniciativas en el seno de la RENAFIPSE van en la misma dirección que las propuestas de la Comisión Ecuatoriana de Nueva Arquitectura Financiera. Se podría imaginar la creación de una red, a nivel de países latinoamericanos, de las diferentes redes nacionales que utilizan medios de pago complementarios. En el futuro, la RENAFIPSE podría articularse con el sistema SUCRE, y más adelante con las acciones del futuro Banco del Sur.

Las potencialidades son numerosas, tanto a nivel local y nacional como regional, siempre con el objetivo de buscar una mayor soberanía económica y financiera que favorezca un mejor desarrollo sostenible, en un marco de integración regional. Se abren nuevas puertas para explorar formas innovadoras de cooperación Sur-Sur, con otros países en desarrollo. Las redes que van construyéndose en torno a estas nuevas instituciones y a estas nuevas lógicas de relaciones, pueden constituir la base para una redefinición de los términos crecimiento y desarrollo, basada en una perspectiva no solo más sostenible, sino sobre todo socialmente más justa. 


\section{Bibliografía}

\section{Textos}

CENAF, Análisis y propuesta para la redefinición de las entidades regionales actualmente establecidas en los pilares de la nueva arquitectura financiera regional, Quito, 2009a.

CENAF, Borrador de la propuesta ecuatoriana para la configuración de un sistema unitario de compensación regional de pagos (SUCRE) con moneda electrónica, Quito, 2009b.

Stiglitz, Joseph. Globalization and Its Discontents, Nueva York: W.W. Norton \& Company, 2002.

Toussaint, Eric. Banque Mondiale: le Coup d'Etat permanent. L'agenda caché $d u$ Consensus de Washington, Lieja-Paris : CADTM-Syllepse, 2006.

Toussaint, Eric. Banque du Sud et nouvelle crise internationale, Lieja-Paris: CADTM-Syllepse, 2008.

Urrutia, Miguel. Perspectivas del Fondo Latinoamericano de Reservas, Santiago de Chile: Cepalc, 2007.

\section{Sitios web:}

〈http://www.aladi.org/ 〉.

〈http://www.bce.fin.ec/〉.

〈http://www.cenaf.com.ec/〉.

〈http:/ / www.comunidadandina.org/ 〉.

〈http:/ / www.fepp.org.ec/〉.

〈https:/ / www.flar.net/ >.

〈http:/ / pachamama.org.ec/ 〉.

〈http:/ / renafipse.ec/ >.

http://www.socialtrade.org/, http://www.stro.org.uy/ >.

〈http:/ / www.sucrealba.org/〉.

Envío 8 de marzo/2011- Aceptación 18 de abril/2011 